\title{
HIF-1a upregulation exerts the antagonistic effect against angiogenesis inhibition in manganese deficiency-induced tibial dyschondroplasia of broiler chicks
}

lu lu

Shandong Agricultural University

Cong Jin

Yishui Animal Disease Control Center

Peng-Fei Dong

Shandong Agricultural University

Lin Wang

Shandong Agricultural University

Zhen-Yong Wang ( $\nabla$ wzy@sdau.edu.cn )

Shandong Agricultural University https://orcid.org/0000-0003-1904-4372

\section{Research Article}

Keywords: Broiler, Manganese, Tibial dyschondroplasia, Angiogenesis, HIF-1a, Autophagy

Posted Date: February 17th, 2022

DOI: https://doi.org/10.21203/rs.3.rs-1353089/v1

License: (c) (i) This work is licensed under a Creative Commons Attribution 4.0 International License.

Read Full License 


\section{Abstract}

Manganese $(\mathrm{Mn})$ is an essential microelement for broiler breeding and its deficiency causes tibial dyschondroplasia (TD). Tibial growth plate (TGP) development and metaphyseal vascularization are crucial for tibia growth in fast-growing broiler chickens, but their roles in Mn deficiency-induced TD in chicks remains unclear. This study was designed to clarify this issue. A total of 36 one-day-old broilers were divided into the control group and Mn-deficiency (Mn-D) group, which were fed with a standard diet (60 mg Mn/ $\mathrm{kg}$ ) and $\mathrm{Mn}$ deficiency diet (22 $\mathrm{mg} \mathrm{Mn} / \mathrm{kg}$ ) for 42 days, respectively. TGP and proximal tibial metaphysis were collected to perform the related assays. First, Mn deficiency decreased the tibia length and TGP thickness in TD model. Mn deficiency reduced the number of blood vessels in proximal tibial metaphysis by histological analysis. Also, Mn deficiency decreased the mRNA and protein expression levels of vascular endothelial growth factor (VEGF) and macrophage migration inhibitory factor (MIF) in TGP. Moreover, up-regulation of hypoxia-inducible factor-1 alpha (HIF-1a) at both mRNA and protein levels was found in TGP of Mn-D group, suggesting the antagonistic role of HIF-1a against angiogenesis inhibition. Additionally, Mn deficiency enhanced the autophagy related 5 (ATG5) and microtubule associated protein 1 light chain 3 beta (LC3-II) expression levels, but decreased the SQSTM1 (p62) expression levels in TGP, suggesting autophagy activation in this process. Collectively, these data indicate that HIF-1 a up-regulation and concurrent autophagy activation exert the protective effect against Mn deficiency-induced angiogenesis inhibition, which may provide a useful guidance to prevent TD in broilers.

\section{Introduction}

Manganese $(\mathrm{Mn})$ is an essential trace element to maintain the normal biological process of livestock and poultry (Liu et al. 2013), particularly in the growth and development of broiler tibia. It has been demonstrated that Mn deficiency diet increases the incidence of osteoporosis and tibial dyschondroplasia (TD) in fast-growing broiler chickens (Zhang et al. 2020). TD, characterized by extensive non-mineralized and avascular cartilage near the tibial growth plate (TGP), is an intractable tibiotarsal bone disorder in fast-growing poultry, which results in the death of tibial chondrocytes due to insufficient or untimely blood supply (Jahejo and Tian 2021; Niu et al. 2021). TD seriously affects the movement of broilers and the welfare of poultry, which causes large economic losses to breeding farms. Previous study has confirmed that developmental disorder of TGP contributes to Mn deficiency-induced TD (Liao et al. 2019), thus this study was designed to investigate the underlying mechanism of Mn deficiency-induced TGP developmental disorder from the perspective of vascularization of proximal metaphyseal tibia.

Angiogenesis is closely associated with osteogenesis (Genin et al. 2012). During the elongation of the tibia, chondrocytes in the TGP resting zone complete longitudinal bone development by proliferation, differentiation, matrix mineralization and apoptosis (Yan et al. 2016). This process is inseparable from the metaphyseal blood vessels, which plays a pivotal role in TGP chondrocyte development, such as mediating the transportation of oxygen, nutrients, and wastes, as well as providing vascular secretion 
signals to control TGP metabolism (Fong et al. 2009). Any defect in the development of metaphyseal vessels may affect the normal differentiation of TGP chondrocytes into bone, subsequently causing TD in broilers (Asmussen et al. 2021). As above mentioned, a well-functioning vascular system is critical for maintaining the normal metabolism and homeostasis of TGP and ensuring leg health of livestock and poultry, but whether Mn deficiency-induced TGP development disorder is related to the abnormal development of metaphyseal vessels remains to be further clarified.

Hypoxia is a representative feature of the TGP micro-environment, which affects angiogenesis in the metaphysis and tibia development (Zhang et al. 2015). Previous study has shown that hypoxia-inducible factor-1 alpha (HIF-1a) is a major modulator of hypoxia response and neovascularization (Im and Kim 2017). Hypoxia induces generation of almost all important angiogenic factors (Dong et al. 2020), such as vascular endothelial growth factor (VEGF) and macrophage migration inhibitory factor (MIF) (Alonso et al. 2019; Zhang et al. 2018). Numerous studies have reported that VEGF is a pro-angiogenic cytokine that sustains angiogenesis (Sojo et al. 2005). MIF is involved in both angiogenesis and cell migration as well as regulation of the angiogenic signaling (Alonso et al. 2019). Besides the direct regulation of angiogenesis, HIF-1 a also regulates angiogenesis by promoting autophagy activation of chondrocytes (Lu et al. 2018). Autophagy is a lysosomal degradation pathway essential for cell survival (Yu et al. 2021), which plays an irreplaceable role in protecting chondrocytes from hypoxia (Singh et al. 2020). Many studies have shown that HIF-1 a mediated autophagy defends against pathological damage of organisms by converting damaged organelles and proteins into energy substances during hypoxia (Zhang et al. 2019). It has also been reported that autophagy activation provides essential amino acids and glucose for TGP angiogenesis under stress conditions to ensure the survival of chondrocytes (Jahejo and Tian 2021). At the same time, enhancing chondrocyte autophagy can also delay the progress of TD by affecting intracellular metabolic activity via regulating cell aging and death (Luo et al. 2019). It is known that the occurrence of TD is attributed to the cellular death of chondrocytes caused by insufficient blood supply, but the mechanisms by which Mn deficiency affects angiogenesis remains to be elucidated.

Therefore, this study employed Mn deficiency-induced broiler TD model to investigate the above unresolved issues. Our results demonstrated that Mn deficiency inhibits metaphysis angiogenesis to prevent the development of the tibia in broiler chicks and HIF-1a may become a novel molecular therapeutic target for improving proximal tibial metaphysis vascularization in TD chicken.

\section{Materials And Methods}

\subsection{Antibodies and reagents}

The following primary antibodies were used: VEGF (A5708), MIF (A1391), and HIF-1a (A11945) were bought from ABclonal Technology Co., Ltd. (Wuhan, China). ATG5 (A0432) was obtained from Abcam (Cambridge Science Park, Cambridge, UK). p62 (PM045) and LC3 (M186-3) were obtained from MBL Co., Ltd. (Nagoya, Japan). ß-actin (GB12001) was purchased from Servicebio Technology Co., Ltd. (Wuhan, 
China). $\beta$-tubulin (66031-1-lg) and GAPDH (10494-1-AP) were obtained from Proteintech Group, Inc. (Wuhan, China). Secondary antibodies used HRP-labeled Goat Anti-Mouse $\lg G(H+L)(C W 1013 A)$ and HRP-labeled Goat Anti-Rabbit IgG(H+L) (CW0102A) from CWBIO biotechnology Co., Ltd. (Beijing, China). RNAiso Plus, Transcriptor cDNA Synth. Kit and SYBR Green I Master were purchased from Roche Co., Ltd (Shanghai, China).

\subsection{Experimental design}

One-day-old healthy commercial Arbor Acres (AA) broilers $(n=36)$ were purchased from New Hope Liuhe Group in Tai'an City, Shandong Province. All chickens were housed in standard wire cages (size, $80 \times 60 \times$ $50 \mathrm{~cm}^{3}$ ), using a recommended standard breeding temperature, humidity and daily lighting. Brooding temperature was maintained at $33^{\circ} \mathrm{C}$ to $35^{\circ} \mathrm{C}$ during the first week and steadily reduced to $29^{\circ} \mathrm{C}$ at the end of second week. During the experiments, the humidity was controlled at around $70 \%$, daily lighting was fixed with $23 \mathrm{~h}$ of light and $1 \mathrm{~h}$ of dark. Additionally, feed and water were provided ad libitum. After 3 days, chickens were randomly divided into two groups $(n=18)$, and each group was given the same basal diet with a different $\mathrm{Mn}$ supplement, i.e., control group (60 mg Mn/kg.feed) and Mn-deficiency (Mn-D) group (22 mg Mn/kg.feed) (Table 1)(Dong et al. 2021). Each group had 3 independent replicates ( $n=6$ per replicate). After $42 \mathrm{~d}$ of feeding, all chickens were sacrificed, and proximal TGP and metaphysis of tibial tissues (approximately $5 \mathrm{~g}$ ) were collected. The length of tibia and the thickness of the TGP were measured with vernier caliper. In addition, metaphysis of 3 broilers in each replicate were put into $4 \%$ formaldehyde fixation solution for histological assessment. TGP of 3 broilers in each replicate were immediately stored in liquid nitrogen and then transferred to $-80{ }^{\circ} \mathrm{C}$ refrigerator for western blot and qRTPCR analysis. All designing schemes in this study conform to animal welfare and have been approved by the Ethic Animal Care Committee of Shandong Agricultural University. 
Table 1

Ingredients and nutrient composition of diets to AA broilers.

\begin{tabular}{|c|c|c|}
\hline Item & \multicolumn{2}{|c|}{ Content } \\
\hline Ingredient & \multicolumn{2}{|c|}{ 1-3week 4-6week } \\
\hline Corn & 60.96 & 67.18 \\
\hline Soybean meal & 10.81 & 10.25 \\
\hline Corn gluten meal & 22.54 & 17.84 \\
\hline Fish meal & 2.00 & 1.00 \\
\hline $\mathrm{CaHPO}_{3}$ & 1.59 & 1.22 \\
\hline Limestone & 1.36 & 1.78 \\
\hline Salt & 0.3 & 0.3 \\
\hline Lysine & 0.34 & 0.33 \\
\hline Microconstituents ${ }^{1}$ & 0.10 & 0.10 \\
\hline Total & 100 & 100 \\
\hline \multicolumn{3}{|l|}{ Nutrient composition \% } \\
\hline Metabolizable energy /(MJ/kg) & 13.39 & 13.39 \\
\hline Crude protein \% & 23.00 & 20.00 \\
\hline Non-phytate phosphorus \% & 1 & 1 \\
\hline Lysine \% & 0.45 & 0.35 \\
\hline Methionine \% & 1.1 & 1 \\
\hline \multicolumn{3}{|c|}{ Nutrient level of the diets was based on NRC recommendations. } \\
\hline \multicolumn{3}{|c|}{$\begin{array}{l}\text { Supplied per kilogram of diet: vitamin A, } 12500 \mathrm{IU} \text {; vitamin D, } 33500 \mathrm{IU} \text {; vitamin E, } 18.75 \mathrm{mg} \text {; vitamin } \mathrm{K} \text {, } \\
32.65 \mathrm{mg} \text {; vitamin B, } 26 \mathrm{mg} \text {; Biotin } 0.0325 \mathrm{mg} \text {, folic acid } 12 \mathrm{mg} \text {, pantothenic acid } 50 \mathrm{mg} \text {; Cu 8mg, Zn } \\
40 \mathrm{mg} \text {, Fe } 80 \mathrm{mg}, \mathrm{I} 0.35 \mathrm{mg} \text {, Se } 0.15 \mathrm{mg} \text {. }\end{array}$} \\
\hline
\end{tabular}

\subsection{Determining Mn concentrations using microwave digestion-ICP-MS}

The contents of Mn in diet, serum and tibia were measured using microwave digestion-ICP-MS. The sample was digested and treated with nitric acid. Then, the obtained samples were quantitatively transferred with polytetrafluoroethylene and diluted with ultrapure water. All conditions and operations are in accordance with the microwave digestion-ICP-MS requirements and Mn levels was determined by the standard mode. 


\subsection{Histological assessment}

Metaphysis samples were fixed in $4 \%$ paraformaldehyde at $4{ }^{\circ} \mathrm{C}$ in PBS, and then decalcified in $10 \%$ EDTA. As referred to the previous method (Chi et al. 2021; Huang et al. 2017), the metaphysis samples were dehydrated, removed, and embedded with ethanol, xylene and paraffin, respectively. Paraffin sections of $5 \mu \mathrm{m}$ thicknesses were cut, stained with hematoxylin and eosin and mounted on Superfrost plus-coated slides for microscopic examination. In addition, the number of blood vessels in metaphysis was determined by Image-Pro Plus 6.0 software.

\subsection{Quantitative Real-Time polymerase chain reaction (qRT- PCR) analysis}

Total RNA of proximal TGP from broiler was extracted with RNAiso Plus and the concentration of extracted RNA was assessed by analyzing A260/A280 ratio using the MicroDrop ${ }^{\circledR}$ spectrophotometer (BIO-DL Corporation, Shanghai, China). The purified RNA ( $2 \mu \mathrm{g}$ per sample) was reverse-transcribed into cDNA by using Transcriptor cDNA Synth. Kit. qPCR reactions were carried out on a Light Cycler 96 (Roche, Basel, Switzerland) with SYBR Green I Master as previously described (Zhao et al. 2021). The housekeeping gene $\beta$-actin was used to normalize the $C t$ value and the $2^{-\Delta \Delta C t}$ method was employed to calculate relative mRNA levels. Primers used in this study are shown in Table 2.

\subsection{Western blotting analysis}

TGP was ground into powder with liquid nitrogen and then put at $4{ }^{\circ} \mathrm{C}$ for $30 \mathrm{~min}$ for lysis in buffer to prepare total protein. The concentration of protein was determined by the bicinchoninic acid assay (Thermo Fisher Scientific, USA). The detailed procedure was referred to the previous study (Liu et al. 2016). Approximately $20 \mu \mathrm{g}$ of protein was separated by SDS-PAGE on $10 \%$ gel of polyacrylamide and then transferred to PVDF membranes (Millipore Corporation, USA). PVDF membrane was blocked in 5\% skim milk for $1.5 \mathrm{~h}$ and then incubated with primary antibody at $4{ }^{\circ} \mathrm{C}$ overnight. The membranes were washed 3 times with TBST (containing $0.1 \%$ Tween 20) for 8 min each time, and then incubated with appropriate secondary antibodies for $50 \mathrm{~min}$ at room temperature. Images were captured with the Chemidoc XRS (Bio-Rad, Marnes-La-Coquette, France) and quantified by Image $\mathrm{J}$ analysis. The density of each band was normalized to its respective loading control. Three independent experiments were carried out for biological replicates.

\subsection{Statistical analysis}

One-way analysis of variance (ANOVA) was performed to analyze the data using the software packages SPSS Statistics 21.0. Values were expressed as the mean \pm SD and statistical significance was set as $p<$ 0.05 and $p<0.01$.

\section{Results}

\subsection{Mn deficiency causes tibial dyschondroplasia in broilers}


This study has established a TD model using fast-growing broiler chickens by feeding with $22 \mathrm{mg} \mathrm{Mn} / \mathrm{kg}$ diet for $42 \mathrm{~d}$, and the actual Mn content in the control group and the Mn-D group was $60.3 \pm 1.02 \mathrm{mg}$ $\mathrm{Mn} / \mathrm{kg}$.feed and $21.8 \pm 1.4 \mathrm{mg} \mathrm{Mn} / \mathrm{kg}$.feed, respectively. Broilers in the Mn-D group gradually displayed a series of typical clinical symptoms on the 30th day, such as dullness, apocleisis, and finally claudication (data not shown). Mn deficiency-induced TD displayed a series of typical clinical symptoms, such as dullness, apocleisis, and lameness (data not shown). The serum Mn content in control group and Mn-D group was $7.58 \pm 1.18 \mu \mathrm{g} / \mathrm{L}$ and $5.58 \pm 1.06 \mu \mathrm{g} / \mathrm{L}$, respectively, and the tibial $\mathrm{Mn}$ content in control group and TD group was $7.28 \pm 1.07 \mu \mathrm{g} / \mathrm{g}$ and $6.09 \pm 0.88 \mu \mathrm{g} / \mathrm{g}$, respectively, which were significantly decreased in Mn-D group. Meanwhile, we found the skeletal deformity of Mn deficiency chicken, especially the proximal tibia distortion and distal tibia expansion (Fig. 1A), and the tibia length (Fig. 1B) and TGP thickness (Fig. 1C) in Mn-D group were obviously shorter $(p<0.05)$ and narrower $(p<0.01)$ than those in control group. Overall, these results suggest that Mn deficiency inhibits the development of tibia in broiler chickens.

\subsection{Mn deficiency inhibits angiogenesis of metaphysis in broilers}

To investigate the difference of vascular distribution between Mn-D group and control group, results of HE staining on the proximal metaphysis of tibial tissues were shown in Fig. 2. In the control group, the distribution of blood vessels was well-proportioned and well-organized, and the columnar structure was well-preserved, part vessels orderly encircling the TGP (Fig. 2A). However, in Mn-D group, the arrangement was irregular and the number was extremely decreased $(p<0.05)$ in the proximal metaphysis region, while the outline of TGP was blurred and the area was reduced (Fig. 2B and C). Then, we analyzed the expression profiles of MIF and VEGF in Mn deficiency-induced TGP tissues. As shown in Fig. 3, qRT-PCR analysis showed that the mRNA expression levels of VEGF and MIF were significantly down-regulated ( $p$ $<0.05$ ) by Mn deficiency (Fig. 3A and B). Also, Mn deficiency significantly decreased $(p<0.05)$ the protein levels of VEGF and MIF (Fig. $3 C$ and D). These results indicate that Mn deficiency-inhibited mRNA and protein expression levels of VEGF and MIF in TGP tissues contributes to angiogenesis inhibition and subsequent disturbed endochondral ossification.

\subsection{Mn deficiency induces HIF-1a up-regulation in TGP of broilers}

To examine the effect of Mn deficiency on the transcriptional levels of HIF-1a in TGP of broilers, we performed qRT-PCR to detect the relative mRNA levels of HIF-1a in two groups. As shown in Fig. 4A, there were significant increases $(p<0.05)$ in expression levels of HIF-1 $a$ in the Mn-D group as compared to the control group. In addition, immunoblot results showed that Mn deficiency remarkably increased the protein level of HIF-1 a (Fig. 4B). These data demonstrate that Mn deficiency induces HIF-1a upregulation in proximal TGP. 


\subsection{Mn deficiency induces the activation of autophagy in TGP of broilers}

To assess the effect of Mn deficiency on autophagy status in proximal TGP of broilers, the mRNA and protein expression profiles of three autophagy marker proteins were analyzed. Compared with the control group, the mRNA and protein expression levels of ATG5 in the Mn-D group were significantly increased ( $p$ $<0.05$ ) (Fig. 5A and D), while mRNA and protein expression levels of p62 in Mn-D group were significantly decreased $(p<0.05)$ (Fig. 5B and E). Moreover, Mn deficiency increased $(p<0.05)$ the LC3-II mRNA expression level and LC3-II protein level (Fig. 5C and F). These data showed that Mn deficiency induces the activation of autophagy in TGP of broilers

\section{Discussion}

TD is the most common leg disease in broilers, and the incidence of TD can be up to $10 \%$ in some chicken farms, which has been the main cause of lameness in poultry raised for meat production (Mehmood et al. 2019). Mn is an essential element for bone growth (Defu Li 2020; Gajula et al. 2011), and its deficiency leads to the failure of endochondral osteogenesis in tibia of fast-growing broiler chickens (Liao et al. 2019). The National Research Council (NRC) recommends a dietary minimum of 60 $\mathrm{mg} \mathrm{Mn} / \mathrm{kg}$ for growing chicks (Mondal et al. 2010). Thus, Mn deficiency diet (21.8 $\pm 1.4 \mathrm{mg} \mathrm{Mn} / \mathrm{kg}$ ) was fed with growing chicks to establish Mn deficiency-induced TD in this study. We found that Mn deficiency inhibits angiogenesis in the proximal tibial metaphysis by down-regulating VEGF and MIF expression levels, while HIF-1 a upregulation and autophagy activation in TGP play a protective role in this process.

During the elongation of the tibia, chondrocytes are transformed into TGP cartilage and bone tissue adjacent to the metaphysis through proliferation and differentiation to increase the tibia length.

Metaphyseal vascularization is closely related to the chondrocyte maturation in TGP and tibial elongation (Herzog et al. 2011). The final event of endochondral ossification is the replacement of avascular cartilage tissue with highly vascularized bone tissue, and the failure of angiogenesis is liable to increase the incidence of TD (Sivaraj and Adams 2016). During this process, MIF and VEGF are secreted by chondrocytes in hypertrophic chondrocyte zone of TGP to induce the vascular invasion and osteogenesis, which play a crucial role in regulate angiogenesis and enhance endothelial cell adhesion, migration, proliferation and microtubule formation (Zhang et al. 2018). In this study, we found that the number of blood vessels in the Mn-D group was sharply reduced, which led to the transportation hurdles of nutrition to chondrocyte. Ultimately, cartilage in the zone of mineralization and calcium deposition fails to complete the calcification. This study showed that Mn deficiency inhibited the metaphysis vascularization and down-regulated the expression levels of VEGF and MIF in TGP of proximal tibia in chicks, which is consistent with the results of thiram-induced TD in chicks (Nabi et al. 2016). Our research group previously found that $\mathrm{Mn}$ deficiency induces an increase in apoptotic death of hypertrophic chondrocytes in TGP (Wang et al. 2015), while the present data further revealed that insufficient blood supply in TGP is another factor contributing to the death of hypertrophic chondrocytes. Given the above 
mentioned, we guess that down-regulation of VEGF and MIF expression in TGP may be responsible for the inhibition of metaphysis vascularization, resulting in inhibition of TGP development, but it needs to be further investigated.

TGP resides in a low oxygen environment and it is an avascular tissue that obtains nutrition and oxygen by diffusion from the metaphysis vascular (Alonso et al. 2019; Sivaraj and Adams 2016). Many previous studies have shown that HIF-1 a, a master regulator of the cellular response to hypoxia, is essential for growth and survival of TGP chondrocytes in vivo (Jahejo and Tian 2021; Wang et al. 2018). HIF-1a is expressed in a hypoxia-independent manner under physiological conditions, mainly in hypertrophic zone of TGP, where it is necessary for chondrocyte maturation and differentiation (Hu et al. 2020) (Lee et al. 2012). It has been shown that inadequate blood supply to chondrocytes leads to a decrease in oxygen concentration and an up-regulation of HIF-1a expression in TGP of chicks with TD (Huang et al. 2018). Moreover, chondrocytes lacking functional HIF-1a undergo massive cell death in the TGP, which leads to the tibia narrow and exhibits less vascularization in metaphysis (Oda et al. 2008). Another hypoxia experiment using AA chicks has shown that tibial angiogenesis is enhanced by upregulation of proangiogenic factors, such as MIF and VEGF, contributing to oxygen and nutrition delivery to TGP (Huang et al. 2017). Data in this study showed that Mn deficiency induces the HIF-1a up-regulation and downregulation of MIF and VEGF expression in TGP. This distinction may be explained that Mn deficiency suppresses normal hypertrophy of chondrocytes in TGP, resulting in decreased production of MIF and VEGF and subsequent inhibition of metaphyseal vascularization. Given the above mentioned, we speculated that HIF-1 a up-regulation may mitigate the chondrocytes damage in TGP caused by Mn deficiency via antagonizing the decrease in angiogenesis.

Autophagy is an evolutionarily conserved process that plays an important role in maintaining intracellular homeostasis and degrading cytoplasmic components, such as damaged organelles and misfolded proteins, to provide energy for the body (Song et al. 2017) (Lu et al. 2018). Recent study has shown that hypoxia can activate autophagy and promote lysosomal degradation of proteins and organelles (Muz et al. 2015). Hypoxia-induced autophagy has been shown to be initiated by HIF-1a in a variety of cell lines (Zhang et al. 2008). In hypoxic condition, the degradation of HIF-1a is inhibited, resulting in increased accumulation of HIF-1a, which directly binds to the hypoxia response elements of the target genes that are involved in various events including angiogenesis, cell migration and tumor invasion (Hu et al. 2012). Previous studies have shown that inhibition of autophagy genes results in the death of chondrocytes, suggesting that autophagy functions as cytoprotective effect in tibial growth (Wang et al. 2021) (Yang et al. 2020). It is reported that HIF-1 a up-regulation can induce the autophagy activation under stress conditions to ensure the survival of TGP chondrocytes (Hu et al. 2020; Singh et al. 2020). Data in this study showed that Mn deficiency induces the autophagy activation of TGP in chicks. This may be ascribed to the decreased angiogenesis in metaphyseal regions, which results in hypoxia environment in TGP and subsequent HIF-1a up-regulation. We speculate that autophagy activation may provide essential energy for chondrocytes and metaphyseal vessels to ensure normal secretion of MIF and VEGF and normal angiogenesis in metaphysis, further indicating that HIF-1 a up-regulation protects against Mn deficiency-induced abnormal metaphysis angiogenesis in broilers. However, the role of autophagy in 
abnormal TGP development and anomalous metaphyseal angiogenesis induced by $\mathrm{Mn}$ deficiency has not been fully elucidated, which needs to be further investigated.

In summary, our findings demonstrate that HIF-1 a up-regulation plays a protective role in Mn deficiencyinduced TGP developmental disorder by antagonizing the angiogenesis inhibition and inducing autophagy activation, which provides us a new insight to the pathogenesis of $\mathrm{Mn}$ deficiency-induced TD.

\section{Declarations}

\section{Acknowledgements}

We would like to thank Professor Rui-Feng Fan for his helpful advice.

\section{Ethics Approval}

All animal experimental protocols were in accordance with the animal welfare and approved by the Ethic Animal Care Committee of Shandong Agricultural University.

\section{Competing Interests}

The authors declare no competing interests.

\section{Contributions}

Lu Lu, conceived of or designed study and performed research. Lu Lu, Cong Jin, and Peng-Fei Dong: Data curation, Writing e original draft \& editing. Lin Wang and Zhen-Yong Wang: Supervision, Resources, Writing review \& editing, Project administration. All authors have read and agreed to the published version of the manuscript.

\section{Data Availability}

The datasets generated during and/or analyzed during the current study are available from the corresponding author on reasonable request

\section{Corresponding author}

Correspondence to Zhen-Yong Wang, E-mail address:wzy@sdau.edu.cn (Wang ZY)

\section{Consent for publication}

We consent to the publication of the submitted manuscript.

Funding This work was supported by grants from the National Natural Science Foundation of China (32072927), Shandong Provincial Natural Science Foundation of China (No. ZR2019MC068), and Youth 
Innovation and Technology Program in Colleges and Universities of Shandong Province (no. 2020KJF009).

\section{References}

1. Alonso D, Serrano E, Bermejo FJ, Corral RS (2019) HIF-1alpha-regulated MIF activation and Nox2dependent ROS generation promote Leishmania amazonensis killing by macrophages under hypoxia. Cell Immunol 335:15-21. DOI: 10.1016/j.cellimm.2018.10.007

2. Asmussen NC, Cohen DJ, Lin Z, McClure MJ, Boyan BD, Schwartz Z (2021) Specific MicroRNAs Found in Extracellular Matrix Vesicles Regulate Proliferation and Differentiation in Growth Plate Chondrocytes. Calcif Tissue Int. DOI: 10.1007/s00223-021-00855-y

3. Chi Q, Zhang Q, Lu Y, Zhang Y, Xu S, Li S (2021) Roles of selenoprotein S in reactive oxygen speciesdependent neutrophil extracellular trap formation induced by selenium-deficient arteritis. Redox Biol 44:102003. DOI: 10.1016/j.redox.2021.102003

4. Defu Li XG, Zhenfang Liu L, Huang Y, Zhou P, Liu L, Qin S, Lin C, Liu Q, Hou L, Li H, Cheng S, Ou Fu, Wei Y, Shen Y, Zou, Xiaobo Yang (2020) \& Association between long-term occupational manganese exposure and bone quality among retired workers. Environ Sci Pollut Res Int 27, 482-489 (2020)

5. Dong J, Chen Y, Yang W, Zhang X, Li L (2020) Antitumor and anti-angiogenic effects of artemisinin on breast tumor xenografts in nude mice. Res Vet Sci 129:66-69. DOI: 10.1016/j.rvsc.2020.01.005

6. Dong PF, Jin C, Lian CY, Wang L, Wang ZY (2021) Enhanced Extracellular Matrix Degradation in Growth Plate Contributes to Manganese Deficiency-Induced Tibial Dyschondroplasia in Broiler Chicks. Biol Trace Elem Res DOI. 10.1007/s12011-021-02921-w

7. Fong L, Tan K, Tran C, Cool J, Scherer MA, Elovaris R, Coyle P, Foster BK, Rofe AM, Xian CJ (2009) Interaction of dietary zinc and intracellular binding protein metallothionein in postnatal bone growth. Bone 44:1151-1162. DOI: 10.1016/j.bone.2009.02.011

8. Gajula SS, Chelasani VK, Panda AK, Mantena VL, Savaram RR (2011) Effect of supplemental inorganic $\mathrm{Zn}$ and $\mathrm{Mn}$ and their interactions on the performance of broiler chicken, mineral bioavailability, and immune response. Biol Trace Elem Res 139:177-187. DOI: 10.1007/s12011-0108647-8

9. Genin O, Hasdai A, Shinder D, Pines M (2012) The effect of inhibition of heat-shock proteins on thiram-induced tibial dyschondroplasia. Poult Sci 91:1619-1626. DOI: 10.3382/ps.2012-02207

10. Herzog A, Genin O, Hasdai A, Shinder D, Pines M (2011) Hsp90 and angiogenesis in bone disorderslessons from the avian growth plate. Am J Physiol Regul Integr Comp Physiol 301:R140-R147. DOI: 10.1152/ajpregu.00134.2011

11. Hu S, Zhang C, Ni L, Huang C, Chen D, Shi K, Jin H, Zhang K, Li Y, Xie L, Fang M, Xiang G, Wang X, Xiao J (2020) Stabilization of HIF-1alpha alleviates osteoarthritis via enhancing mitophagy. Cell Death Dis 11:481. DOI: 10.1038/s41419-020-2680-0 
12. Hu YL, DeLay M, Jahangiri A, Molinaro AM, Rose SD, Carbonell WS, Aghi MK (2012) Hypoxia-induced autophagy promotes tumor cell survival and adaptation to antiangiogenic treatment in glioblastoma. Cancer Res 72:1773-1783. DOI: 10.1158/0008-5472.CAN-11-3831

13. Huang S, Wang M, Rehman MU, Zhang L, Tong X, Shen Y, Li J (2018) Role of Angiopoietin-like 4 on Bone Vascularization in Chickens Exposed to High-altitude Hypoxia. J Comp Pathol 161:25-33. DOI: 10.1016/j.jcpa.2018.04.007

14. Huang SC, Rehman MU, Lan YF, Qiu G, Zhang H, Iqbal MK, Luo HQ, Mehmood K, Zhang LH, Li JK (2017) Tibial dyschondroplasia is highly associated with suppression of tibial angiogenesis through regulating the HIF-1alpha/VEGF/VEGFR signaling pathway in chickens. Sci Rep 7:9089. DOI: $10.1038 /$ s41598-017-09664-6

15. Im S, Kim DW (2017) Nkx3.2 induces oxygen concentration-independent and lysosome-dependent degradation of HIF-1alpha to modulate hypoxic responses in chondrocytes. Cell Signal 36:127-138. DOI: 10.1016/j.cellsig.2017.05.001

16. Jahejo AR, Tian WX (2021) Cellular, molecular and genetical overview of avian tibial dyschondroplasia. Res Vet Sci 135:569-579. DOI: 10.1016/j.rvsc.2020.10.002

17. Lee SH, Che X, Jeong JH, Choi JY, Lee YJ, Lee YH, Bae SC, Lee YM (2012) Runx2 protein stabilizes hypoxia-inducible factor-1alpha through competition with von Hippel-Lindau protein (pVHL) and stimulates angiogenesis in growth plate hypertrophic chondrocytes. J Biol Chem 287:14760-14771. DOI: $10.1074 / j b c . M 112.340232$

18. Liao XD, Wang G, Lu L, Zhang LY, Lan YX, Li SF, Luo XG (2019) Effect of manganese source on manganese absorption and expression of related transporters in the small intestine of broilers. Poult Sci 98:4994-5004. DOI: 10.3382/ps/pez293

19. Liu G, Wang ZK, Wang ZY, Yang DB, Liu ZP, Wang L (2016) Mitochondrial permeability transition and its regulatory components are implicated in apoptosis of primary cultures of rat proximal tubular cells exposed to lead. Arch Toxicol 90:1193-1209. DOI: 10.1007/s00204-015-1547-0

20. Liu XF, Zhang LM, Zhang Z, Liu N, Xu SW, Lin HJ (2013) Manganese-induced effects on testicular trace element levels and crucial hormonal parameters of Hyline cocks. Biol Trace Elem Res 151:217224. DOI: $10.1007 / \mathrm{s} 12011-012-9549-8$

21. Lu N, Li X, Tan R, An J, Cai Z, Hu X, Wang F, Wang H, Lu C, Lu H (2018) HIF-1alpha/Beclin1-Mediated Autophagy Is Involved in Neuroprotection Induced by Hypoxic Preconditioning. J Mol Neurosci 66:238-250. DOI: 10.1007/s12031-018-1162-7

22. Luo P, Gao F, Niu D, Sun X, Song Q, Guo C, Liang Y, Sun W (2019) The Role of Autophagy in Chondrocyte Metabolism and Osteoarthritis: A Comprehensive Research Review. Biomed Res Int 2019:5171602. DOI: 10.1155/2019/5171602

23. Mehmood K, Zhang H, Jiang X, Yao W, Tong X, Iqbal MK, Rehman MU, Iqbal M, Waqas M, Qamar H, Zhang J, Li J (2019) Ligustrazine recovers thiram-induced tibial dyschondroplasia in chickens: Involvement of new molecules modulating integrin beta 3. Ecotoxicol Environ Saf 168:205-211. DOI: 10.1016/j.ecoenv.2018.10.080 
24. Mondal S, Haldar S, Saha P, Ghosh TK (2010) Metabolism and tissue distribution of trace elements in broiler chickens' fed diets containing deficient and plethoric levels of copper, manganese, and zinc. Biol Trace Elem Res 137:190-205. DOI: 10.1007/s12011-009-8570-z

25. Muz B, de la Puente P, Azab F, Azab AK (2015) The role of hypoxia in cancer progression, angiogenesis, metastasis, and resistance to therapy. Hypoxia (Auckl) 3:83-92. DOI: 10.2147/HP.S93413

26. Nabi F, Shahzad M, Liu J, Li K, Han Z, Zhang D, Iqbal MK, Li J (2016) Hsp90 inhibitor celastrol reinstates growth plate angiogenesis in thiram-induced tibial dyschondroplasia. Avian Pathol 45:187-193. DOI: 10.1080/03079457.2016.1141170

27. Niu S, Li X, Jahejo AR, Zhang N, Yang SX, Jia YF, Zhang YY, Tian ZX, Li Z, Ning GB, Zhang D, Tian WX (2021) Glutathione-S-transferase A3 protein suppresses thiram-induced tibial dyschondroplasia by regulating prostaglandin-related genes expression. Res Vet Sci 135:343-348. DOI:

10.1016/j.rvsc.2020.10.014

28. Oda S, Oda T, Nishi K, Takabuchi S, Wakamatsu T, Tanaka T, Adachi T, Fukuda K, Semenza GL, Hirota K (2008) Macrophage migration inhibitory factor activates hypoxia-inducible factor in a p53dependent manner. PLoS ONE 3:e2215. DOI: 10.1371/journal.pone.0002215

29. Singh A, Wilson JW, Schofield CJ, Chen R (2020) Hypoxia-inducible factor (HIF) prolyl hydroxylase inhibitors induce autophagy and have a protective effect in an in-vitro ischaemia model. Sci Rep 10:1597. DOI: 10.1038/s41598-020-58482-w

30. Sivaraj KK, Adams RH (2016) Blood vessel formation and function in bone. Development 143:27062715. DOI: $10.1242 /$ dev. 136861

31. Sojo K, Sawaki Y, Hattori H, Mizutani H, Ueda M (2005) Immunohistochemical study of vascular endothelial growth factor (VEGF) and bone morphogenetic protein-2, -4 (BMP-2, -4) on lengthened rat femurs. J Craniomaxillofac Surg 33:238-245. DOI: 10.1016/j.jcms.2005.02.004

32. Song XB, Liu G, Liu F, Yan ZG, Wang ZY, Liu ZP, Wang L (2017) Autophagy blockade and lysosomal membrane permeabilization contribute to lead-induced nephrotoxicity in primary rat proximal tubular cells. Cell Death Dis 8:e2863. DOI: 10.1038/cddis.2017.262

33. Wang B, Shi Y, Chen J, Shao Z, Ni L, Lin Y, Wu Y, Tian N, Zhou Y, Sun L, Wu A, Hong Z, Wang X, Zhang $X$ (2021) High glucose suppresses autophagy through the AMPK pathway while it induces autophagy via oxidative stress in chondrocytes. Cell Death Dis 12:506. DOI: 10.1038/s41419-02103791-9

34. Wang H, Huo X, Chen H, Li B, Liu J, Ma W, Wang X, Xie K, Yu Y, Shi K (2018) Hydrogen-Rich Saline Activated Autophagy via HIF-1alpha Pathways in Neuropathic Pain Model. Biomed Res Int 2018:4670834. DOI: 10.1155/2018/4670834

35. Wang J, Wang ZY, Wang ZJ, Liu R, Liu SQ, Wang L (2015) Effects of manganese deficiency on chondrocyte development in tibia growth plate of Arbor Acres chicks. J Bone Miner Metab 33:23-29. DOI: $10.1007 / \mathrm{s} 00774-014-0563-0$ 
36. Yan B, Zhang Z, Jin D, Cai C, Jia C, Liu W, Wang T, Li S, Zhang H, Huang B, Lai P, Wang H, Liu A, Zeng C, Cai D, Jiang Y, Bai X (2016) mTORC1 regulates PTHrP to coordinate chondrocyte growth, proliferation and differentiation. Nat Commun 7:11151. DOI: 10.1038/ncomms11151

37. Yang H, Wen Y, Zhang M, Liu Q, Zhang H, Zhang J, Lu L, Ye T, Bai X, Xiao G, Wang M (2020) MTORC1 coordinates the autophagy and apoptosis signaling in articular chondrocytes in osteoarthritic temporomandibular joint. Autophagy 16:271-288. DOI: 10.1080/15548627.2019.1606647

38. Yu ZM, Wan XM, Xiao M, Zheng C, Zhou XL (2021) Puerarin induces Nrf2 as a cytoprotective mechanism to prevent cadmium-induced autophagy inhibition and NLRP3 inflammasome activation in AML12 hepatic cells. J Inorg Biochem 217:111389. DOI: 10.1016/j.jinorgbio.2021.111389

39. Zhang F, Liu XL, Wang W, Dong HL, Xia YF, Ruan LP, Liu LP (2018) Expression of MMIF, HIF-1alpha and VEGF in Serum and Endometrial Tissues of Patients with Endometriosis. Curr Med Sci 38:499504. DOI: $10.1007 / \mathrm{s} 11596-018-1906-1$

40. Zhang FJ, Luo W, Lei GH (2015) Role of HIF-1alpha and HIF-2alpha in osteoarthritis. Joint Bone Spine 82:144-147. DOI: 10.1016/j.jbspin.2014.10.003

41. Zhang H, Bosch-Marce M, Shimoda LA, Tan YS, Baek JH, Wesley JB, Gonzalez FJ, Semenza GL (2008) Mitochondrial autophagy is an HIF-1-dependent adaptive metabolic response to hypoxia. $J$ Biol Chem 283:10892-10903. DOI: 10.1074/jbc.M800102200

42. Zhang H, Wang Y, Mehmood K, Chang YF, Tang Z, Li Y (2020) Treatment of tibial dyschondroplasia with traditional Chinese medicines: "Lesson and future directions". Poult Sci 99:6422-6433. DOI: 10.1016/j.psj.2020.08.055

43. Zhang Y, Liu D, Hu H, Zhang P, Xie R, Cui W (2019) HIF-1 alpha/BNIP3 signaling pathway-inducedautophagy plays protective role during myocardial ischemia-reperfusion injury. Biomed Pharmacother 120:109464. DOI: 10.1016/j.biopha.2019.109464

44. Zhao Y, Li ZF, Zhang D, Wang ZY, Wang L (2021) Quercetin alleviates Cadmium-induced autophagy inhibition via TFEB-dependent lysosomal restoration in primary proximal tubular cells. Ecotoxicol Environ Saf 208:111743. DOI: 10.1016/j.ecoenv.2020.111743

\section{Figures}

\section{Figure 1}

Mn deficiency induces TD in broilers. (A) Representative image showing the tibia morphology in the control and Mn-D broilers. (B) Quantitative analysis of the length of tibia in two groups. (C) Quantitative analysis of the thickness of TGP in two groups. Data were expressed as mean $\pm S D(n=18)$. Compared with the control, ${ }^{*} p<0.05 ;{ }^{* *} p<0.01$. 
A

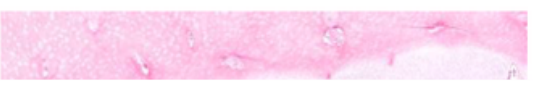

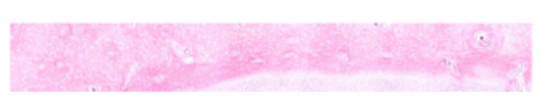

B

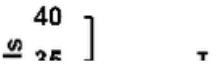

\section{Figure 2}

Mn deficiency inhibits angiogenesis of proximal tibial metaphysis in broilers. (A) Representative image showing the vascular distribution in proximal tibial metaphysis of broilers. The arrows indicated the blood vessels. (B) Numbers of blood vessels were quantified using Image-Pro Plus 6.0 software. Data were expressed as mean $\pm S D$ ( $n=3$ of each independent replicate). Compared with the control, ${ }^{*} p<0.05$; ${ }^{* *} p<0.01$. 

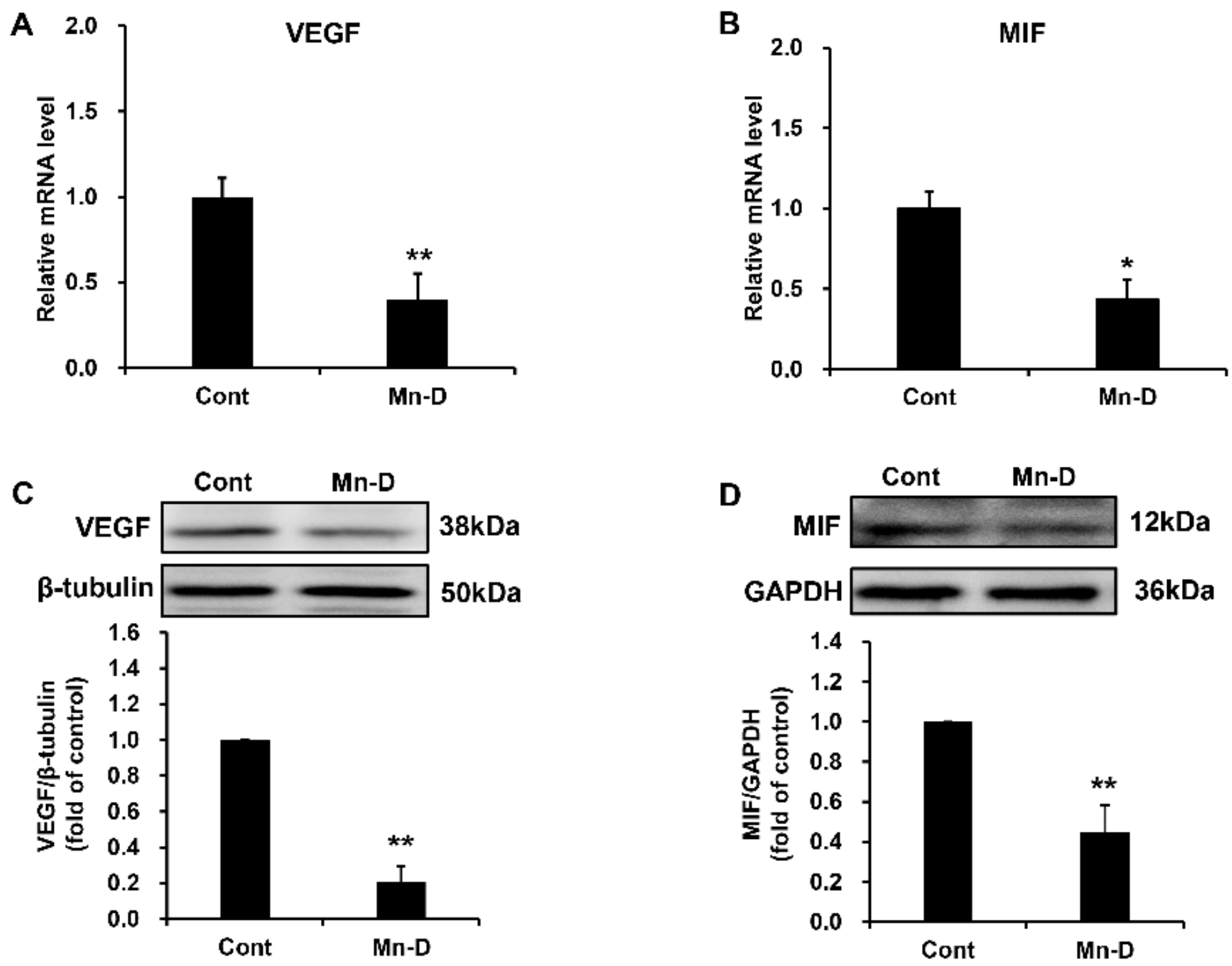

Figure 3

Figure 3

Mn deficiency decreases MIF and VEGF gene and protein expression levels in TGP of broilers. $(A, B)$ mRNA expression levels of VEGF and MIF were determined by qRT-PCR assay. (C, D) Protein levels of VEGF and MIF were assessed by western blot analysis and the results were quantified and analyzed using Image-Pro Plus 6.0. Data were expressed as mean \pm SD ( $n=3$ of each independent replicate). Compared with the control, ${ }^{\star} p<0.05 ; * \star p<0.01$. 
A

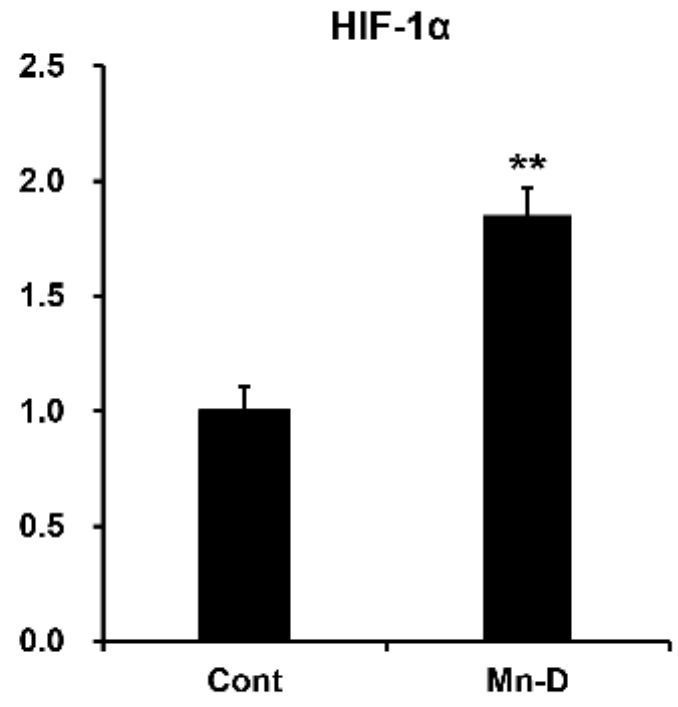

B
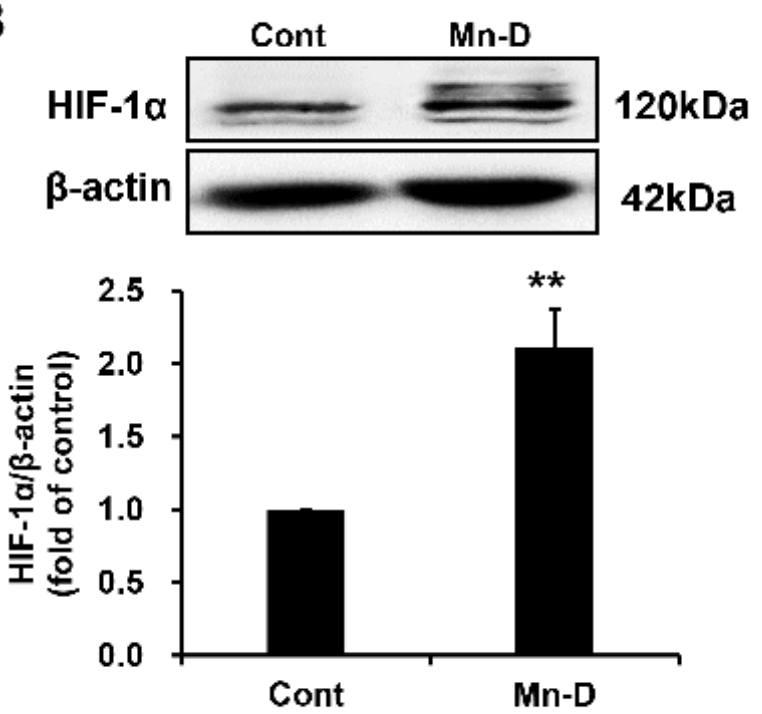

\section{Figure 4}

Figure 4

Mn deficiency enhances HIF-1a mRNA and protein expression levels in TGP of broilers. (A) mRNA expression levels of HIF-1a were determined by qRT-PCR assay. (B) Protein levels of HIF-1a were determined by western blotting analysis and the results were quantified and analyzed using Image-Pro Plus 6.0. Data were expressed as mean $\pm S D$ ( $n=3$ of each independent replicate). Compared with the control, * $p<0.05 ; * \star p<0.01$.

\section{Figure 5}

The expression profiles of ATG5, LC3-II, and p62 in TGP of broilers. (A-C) mRNA expression levels of ATG5, p62 and LC3-II in TGP of broilers were determined by qRT-PCR analysis. (D-F) Protein levels of ATG5, p62 and LC3-II were assessed by immunoblot assay and the results were quantified and analyzed using Image-Pro Plus 6.0. Data were expressed as mean \pm SD ( $n=3$ of each independent replicate). Compared with the control, ${ }^{\star} p<0.05 ; * \star p<0.01$. 